\title{
Analysis of QRS Loop in the Vectorcardiogram of Patients with Chagas' Disease
}

\author{
Raúl Correa, Student Member, IEEE, Eric Laciar, Member, IEEE, \\ Pedro Arini, Member, IEEE, and Raimon Jané, Member, IEEE
}

\begin{abstract}
In the present work, we have studied the QRS loop in the Vectorcardiogram (VCG) of 95 chronic chagasic patients classified in different groups (I, II and III) according to their degree of myocardial damage. For comparison, the VCGs of 11 healthy subjects used as control group (Group O) were also examined. The QRS loop was obtained for each patient from the XYZ orthogonal leads of their HighResolution Electrocardiogram (HRECG) records. In order to analyze the variations of QRS loop in each detected beat, it has been proposed in this study the following vectorcardiographic parameters a) Maximum magnitude of the cardiac depolarization vector, b) Volume, c) Area of QRS loop, d) Ratio between the Area and Perimeter, e) Ratio between the major and minor axes of the QRS loop and f) QRS loop Energy. It has been found that one or more indexes exhibited statistical differences $(p<0.05)$ between groups 0 -II, O-III, I-II, I-III and II-III. We concluded that the proposed method could be use as complementary diagnosis technique to evaluate the degree of myocardial damage in chronic chagasic patients.
\end{abstract}

\section{INTRODUCTION}

$\mathrm{C}$ 'Hagas' disease (American Trypanosomiasis) is a tropical illness caused by a parasite, Trypanosoma Cruzi, which is transmitted commonly by a blood-sucking triatomine bug. This illness is the major cause of cardiac disease in South and Central America. It is estimated that 16 to 18 million Latin Americans have been infected and that 2-3 million may have already developed chronic complications [1].

There are two stages of the disease: the acute stage which appears shortly after the infection, and the chronic stage which appears after a silent and asymptomatic period that may last 10-20 years [2]. The chronic phase is characterized by a progressive inflammation of cardiac muscle (Chagasic myocarditis) that produces a destruction of cardiac fibers and a fibrosis in multiple areas of the myocardium and a malfunctioning in the propagation of the cardiac electrical

This work has been supported by Consejo Nacional de Investigaciones Científicas y Técnicas (CONICET) and Universidad Nacional de San Juan (UNSJ), both institutions from Argentina, and by Ministerio de Ciencia e Innovación de España (TEC2007-68076-C02-01).

R. Correa and E. Laciar are with Gabinete de Tecnología Médica, Facultad de Ingeniería, Universidad Nacional. de San Juan, Argentina. E-mail: rcorrea@gateme.uns.edu.ar, laciar@gateme.unsj.edu.ar

P. Arini is with Instituto Argentino de Matemática, CONICET, Buenos Aires, Argentina. E-mail: pedro.arini@conicet.gov.ar

R. Jané is with Dept. ESAII, Universitat Politècnica de Catalunya, Institut de Bioenginyeria de Catalunya (IBEC) and CIBER de Bioingeniería, Biomateriales y Nanomedicina (CIBER-BBN), Barcelona, España. E-mail: raimon.jane@upc.edu. impulse [3]. This cardiac damage usually has slow, progressive and irreversible characteristics and the symptoms appear when the damage is significant. In order to establish the correct treatment in chronic chagasic patients, it is necessary to determine previously the degree of myocardial damage produced by the disease. Currently, this clinical evaluation is made by different diagnostic procedures, such as chest X-ray, conventional Electrocardiogram (ECG), Vectorcardiogram (VCG), ambulatory ECG (Holter), cardiopulmonary metabolic exercise test, High-Resolution ECG (HRECG), between others [4].

Recently, Perez Riera et al. [5] have exposed in a review the advantages of the computerized VCG compared to the ECG. Particularly, they have been established the higher specificity, sensitivity and accuracy of this technique compared with conventional ECG for the diagnosis of different cardiac pathologies. VCG is defined as the graphic record of the direction and magnitude of the characteristic electric forces of heart activity during a cardiac cycle. In a previous work, we analyzed the dynamic changes of QRS loop in ischemic patients undergoing angioplasty [6] and we observed that the dynamic changes in the VCG during ventricular depolarization reflect the level modifications of cardiac ischemia induced by Percutaneous Transluminal Coronary Angioplasty (PTCA). Ribeiro and Rocha [7] have reported significant cardiovascular abnormalities in 68 to $88 \%$ of abnormal studies of chagasic patients using VCG, including changes in ventricular repolarization, left ventricular hypertrophy, disorders of the final conduction, electrically inactive areas, left anterior hemiblock and diminishing strength septal.

In this study, we propose the analysis of the 3-D Vectorcardiogram (VCG) in order to evaluate the cardiac differences in chronic chagasic patients with diverse cardiac damage. The VCG constructed from XYZ orthogonal leads during each QRS complex are analyzed. The changes of QRS loop are statistically evaluated in each detected beat by six 3-D QRS loop parameters. In this way, the aim of this study is to analyze the ability of these parameters to describe the degree of myocardial damage in chagasic patients.

\section{MATERials}

We analyzed the HRECG of 95 chronic chagasic patients and 11 healthy subjects. All HRECG were recorded during 
10 minutes according to the accepted standard [8], using orthogonal XYZ leads. The sampling rate was $1000 \mathrm{~Hz}$ and the $\mathrm{A} / \mathrm{D}$ resolution was 16 bits. All data were extracted from Chagas Database of the Simon Bolivar University, Venezuela [9].

Subjects were clinically classified into 4 groups, as follow:

- Group $O$ composed of 11 healthy subjects, blood seronegative.

- Group I consisted of 41 chagasic patients, blood seropositive, but no echocardiographic or standard ECG evidence of myocardial damage.

- Group II included 38 chagasic patients, blood seropositive, with some evidence of cardiac involvement detected in 24 hours Holter monitoring.

- Group III included 16 chagasic patients, blood seropositive, with evidences of severe myocardial damage observed in conventional ECG and documented episodes of ventricular tachycardia.

\section{MethodS}

Different stages of analysis are proposed (see block diagram in Fig. 1): Preprocessing, QRS loop estimation, QRS loop alignment and Parameter computations.

\section{A. Preprocessing}

All HRECG records were preprocessed with a notch filter (Butterworth, 4th order, $60 \mathrm{~Hz}$, bidirectional filter) in order to minimize the powerline interference and with a low-pass filter (Butterworth, 8th order, $100 \mathrm{~Hz}$, bidirectional filter) to reduce high frequency noise. An additional filter, based on cubic spline interpolation, was used in order to attenuate ECG baseline drifts and respiratory artifacts. After filtering, the QRS complexes and their corresponding endpoints were detected in each HRECG record using a modified algorithm of the QRS detector proposed by Pan and Tompkins [10].

\section{B. QRS loop Estimation}

The QRS loop is obtained by drawing simultaneously, in

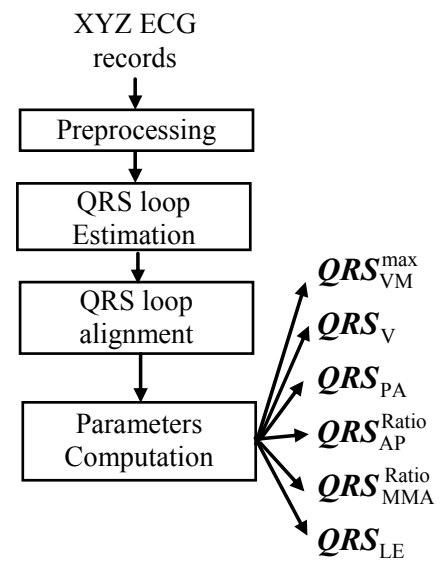

Fig.1. General diagram of the proposed technique for the analysis of QRS loop changes a 3-D plot, the instantaneous amplitudes of XYZ orthogonal leads in the temporal interval corresponding to QRS complex. This interval is defined from the starting point of $\mathrm{Q}$ wave (or R if there is not Q) to the final point of $\mathrm{S}$ wave (or R if there is not $\mathrm{S}$ ).

\section{QRS loop alignment}

In order to analyze the beat-to-beat variations of QRS loops, it is previously necessary to align them. The spatial alignment of QRS loops compensates the changes in the orientation of the cardiac electrical axis caused by various extracardiac factors, like the respiratory induced movements of the heart [11].

The QRS spatial alignment problem can be resolved by obtaining the Rotation (R) and Translation (T) Matrices that allow to align the QRS loop of each beat in HRECG record with respect to a QRS loop template obtained as the VCG of the signal-averaged beat of this record.

In this work, $\mathrm{R}$ and $\mathrm{T}$ matrices were obtained by using the algorithm proposed by Arun et al. [12]. It is based on Singular Value Decomposition (SVD) and gives a closed solution in the computation of both matrices.

\section{Parameters Computation}

The following parameters were computed from the VCG corresponding to QRS loop for each detected beat in HRECG record:

1) Maximum Magnitude of the Depolarization Vector $\left(\boldsymbol{Q R} \boldsymbol{S}_{\mathrm{VM}}^{\max }\right)$ : In order to find it, the vector magnitude for each coordinate $(\mathrm{X}, \mathrm{Y}, \mathrm{Z})$ of $\mathrm{QRS}$ loops is initially calculated, and then the maximum value is obtained [13]. This parameter describes the maximum magnitude of the Depolarization Vector (see Fig. 2).

2) Volume $\left(\boldsymbol{Q R} \boldsymbol{S}_{\mathrm{V}}\right)$ : In order to achieve a more accurate estimation of the volume of the 3-D VCG representation, it has been calculated the set of points that produce the minimum convex volume (using the "Convex Hull" technique [14]), and that contain all points of VCG loop. Then, the volume of this convex figure is evaluated.

3) Planar Area of QRS loop ( $\left.\boldsymbol{Q R} \boldsymbol{S}_{\mathrm{PA}}\right)$ : It is the estimated area of the loop obtained by projecting the QRS loop on the Optimum Plane (OP), which is the best adjusted plane computed by least mean squares. It is thought to reflect hemodynamic abnormalities in cardiac lesions [15]

4) Ratio between the Area and Perimeter ( $\left.\boldsymbol{Q R} \boldsymbol{S}_{\mathrm{AP}}^{\text {Ratio }}\right)$ : This ratio is evaluated over the QRS loop projected in the OP. This parameter was calculated with the aim to analyze changes in the morphology of the QRS loop.

5) Ratio between the Major and Minor Axes ( $\left.\boldsymbol{Q R S _ { \mathrm { MMA } } ^ { \mathrm { Ratio } }}\right)$ : This ratio was also evaluated over the QRS loop projected in the OP and was computed to assess the changes in the morphology of the QRS loop (See Fig. 2). 
TABLE I

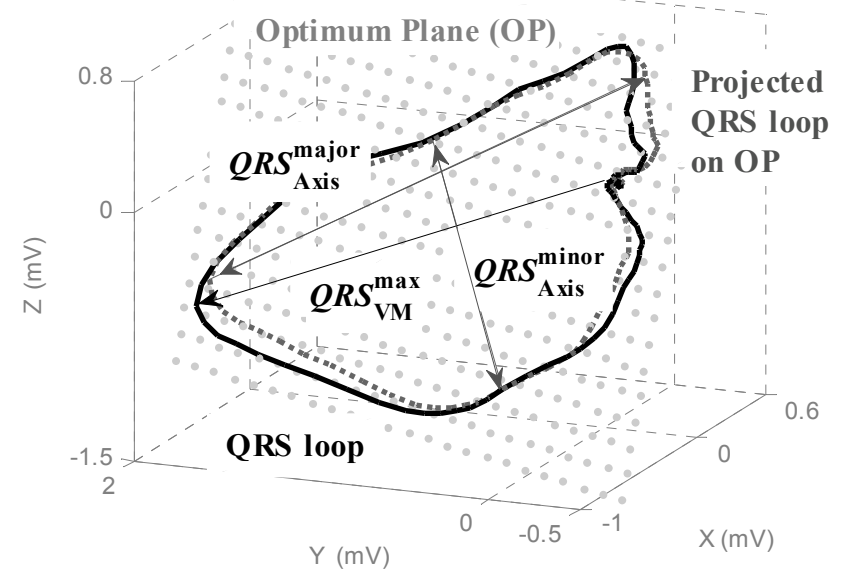

Fig.2. Some characteristic QRS loop parameters for the HRECG recording of the patient \# 2 of the Group: 0

6) QRS Loop Energy $\left(\boldsymbol{Q R S _ { \mathrm { LE } }}\right)$ : In order to assess this parameter, we estimated the RMS value of the XYZ coordinates of QRS loop.

\section{RESUlts}

The described analysis technique was applied to the HRECG records of the 95 chronic chagasic patients (Groups I, II and III) and the 11 healthy subjects used as control group (Group O).

Figure 3 shows graphically the mean value of each QRS loop parameters for the population of healthy subject (Group O) and chagasic patients (Group I, II, III). The top row of this figure (Fig. 3.a -3.f) illustrates the results of $\boldsymbol{Q R} \boldsymbol{S}_{\mathrm{MV}}^{\max }$,

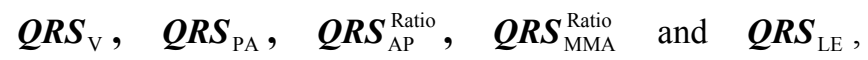
respectively, for each subject of all groups. The bottom row (Fig. 3.g - 3 1) shows the Mean and Standard Error of the (a)

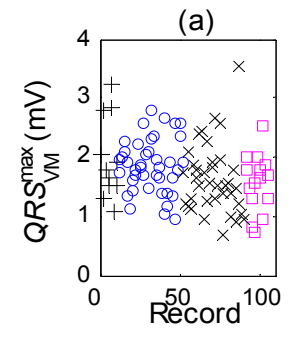

(g)

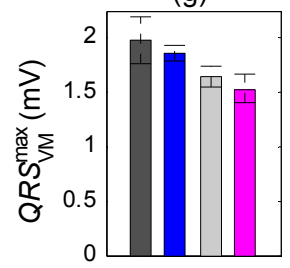

(b)

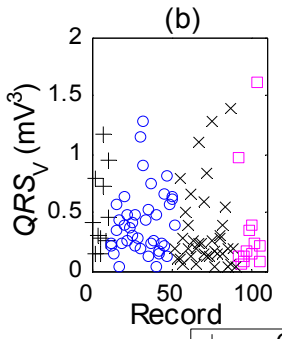

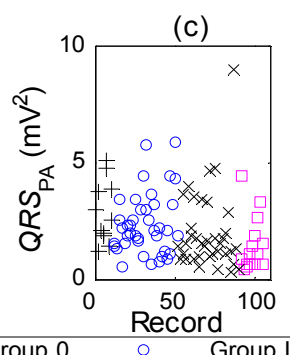

(c)

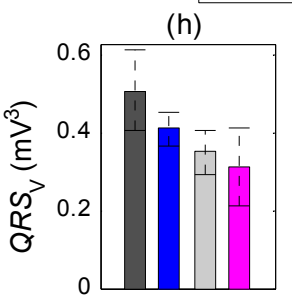

(i)

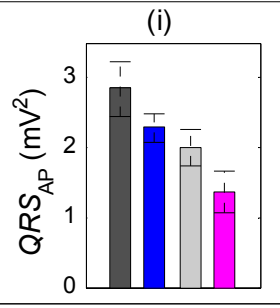

MEAN VALUES \pm SEM FOR EACH PARAMETER FOR EACH GROUP

\begin{tabular}{lcccc}
\hline \hline Parameter & Group 0 & Group I & Group II & Group III \\
\hline $\boldsymbol{Q} R \boldsymbol{S}_{\mathrm{VM}}^{\max }$ & $1,97 \pm 0,21$ & $1,85 \pm 0,07$ & $1,64 \pm 0,09$ & $1,53 \pm 0,12$ \\
$\boldsymbol{Q} \boldsymbol{R} \boldsymbol{S}_{\mathrm{V}}$ & $0,51 \pm 0,10$ & $0,41 \pm 0,04$ & $0,35 \pm 0,06$ & $0,31 \pm 0,10$ \\
$\boldsymbol{Q} \boldsymbol{R} \boldsymbol{S}_{\mathrm{PA}}$ & $2,83 \pm 0,40$ & $2,28 \pm 0,20$ & $1,98 \pm 0,26$ & $1,36 \pm 0,29$ \\
$\boldsymbol{Q R S}_{\mathrm{AP}}^{\text {Ratio }}$ & $2,63 \pm 0,20$ & $3,10 \pm 0,16$ & $3,74 \pm 0,26$ & $4,64 \pm 0,47$ \\
$\boldsymbol{Q R S}_{\mathrm{MMA}}^{\text {Ratio }}$ & $2,23 \pm 0,16$ & $2,90 \pm 0,22$ & $3,05 \pm 0,25$ & $4,53 \pm 0,59$ \\
$\boldsymbol{Q R S}_{\mathrm{LE}}$ & $0,49 \pm 0,05$ & $0,44 \pm 0,02$ & $0,39 \pm 0,02$ & $0,36 \pm 0,03$ \\
\hline
\end{tabular}

Mean (SEM) values of each parameter for all subjects of each group. These last values are quantified in Table I.

The Kolmogorov - Smirnov test was applied in order to quantify the discrepancy between the vectorcardiographic parameters distribution and an ideal Gaussian distribution. It has been found that vectorcardiographic parameters do not follow a Gaussian distribution. Afterwards, comparisons between groups were made using a non-parametric MannWhitney test. Table II presents the results of $p$-values of the TABLE II

P-VALUES OF MANN-WHITNEY STATISTICAL TEST FOR EACH INDEX WHEN COMPARING TWO DIFFERENT GROUPS

\begin{tabular}{lcccccc}
\hline \hline \multirow{2}{*}{ Parameter } & \multicolumn{5}{c}{ Groups } \\
\cline { 2 - 7 } & O vs I & O vs II & O vs III & I vs II & I vs & II vs III \\
\hline $\boldsymbol{Q R S}_{\mathrm{VM}}^{\text {max }}$ & 0,884 & 0,144 & 0,186 & 0,022 & 0,051 & 0,906 \\
$\boldsymbol{Q R S}_{\mathrm{V}}$ & 0,413 & 0,055 & 0,036 & 0,062 & 0,026 & 0,515 \\
$\boldsymbol{Q R S}_{\mathrm{PA}}$ & 0,198 & 0,018 & 0,005 & 0,088 & 0,008 & 0,089 \\
$\boldsymbol{Q R S}_{\mathrm{AP}}^{\text {Ratio }}$ & 0,175 & 0,013 & 0,005 & 0,064 & 0,006 & 0,139 \\
$\boldsymbol{Q R S}_{\mathrm{MMA}}^{\text {Ratio }}$ & 0,136 & 0,033 & 0,009 & 0,372 & 0,017 & 0,048 \\
$\boldsymbol{Q R S}_{\mathrm{LE}}$ & 0,662 & 0,055 & 0,036 & 0,035 & 0,018 & 0,693 \\
\hline
\end{tabular}
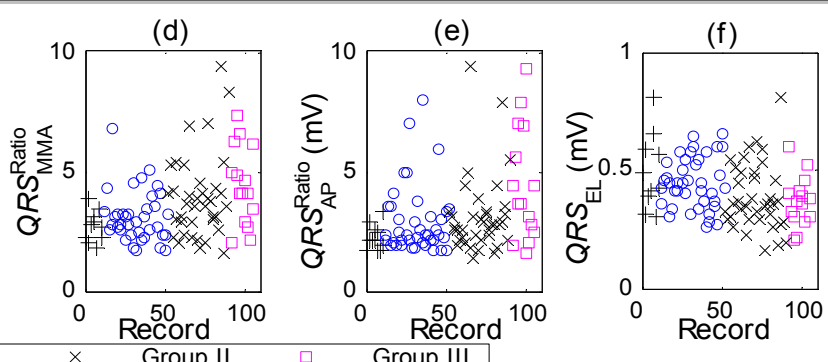

(j) (k)
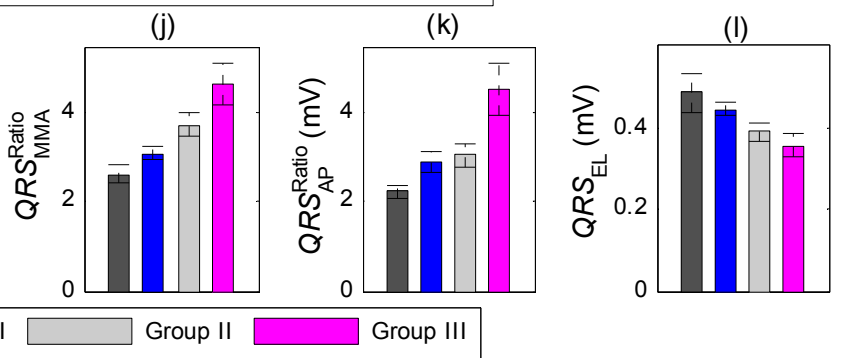

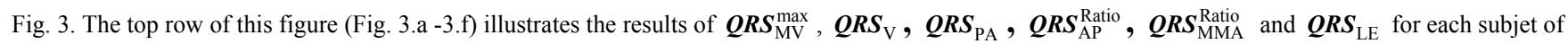
all groups. The bottom row (Fig. 3.g - 3 l) shows the mean and Standard Error of the Mean (SEM) values of each parameter for all subjects of each group. 
Mann-Whitney statistical test for each index when comparing two different groups. The p-values which are statistically significant $(p<0.05)$ have been indicated on the table with gray shading.

\section{DisCUSSION AND CONCLUSIONS}

Some works have proposed the use of the VCG technique for the study and diagnosis of the Chagas' disease [4], [16], based on the VCG 2-D projection on the Frontal, Horizontal or Sagittal planes. In this study, we have examined the QRS loop changes using a 3-D representation in 95 chagasic patients with different cardiac damage (Groups I, II and III) and 11 healthy subjects used as control group (Group O) Also, we have proposed six vectorcardiographic parameters in order to assess the QRS loop changes in this 3-D scheme.

The results shown in Fig. 3 and Table I indicate that the mean value of $\boldsymbol{Q R} \boldsymbol{S}_{\mathrm{AP}}^{\mathrm{Ratio}}$ and $\boldsymbol{Q R} \boldsymbol{S}_{\mathrm{MMA}}^{\text {Ratio }}$ parameters increased progressively from Group $\mathrm{O}$ to Group III, according to Chagas' disease severity. By contrast, the mean value of $\boldsymbol{Q R} \boldsymbol{S}_{\mathrm{V}}$ and $\boldsymbol{Q R} \boldsymbol{S}_{\mathrm{PA}}$, decreased as the cardiac damage produced by the disease increases. These results agree with previous works of our group, using different parameters, obtained from signal-averaged and beat-to-beat analysis of HRECG [17] and QRS slopes [18].

The statistical analysis shown in Table 2 reveals that one or more parameters exhibit statistical differences $(\mathrm{p}<0.05)$ between the groups O-II, O-III, I-II, I-III and II-III. However, none of the parameters indicate statistical differences between groups $\mathrm{O}$ and $\mathrm{I}$. This last result is in agreement with medical practice and previous works of our group [17], [18], since the chagasic patients of the group I do not present any diagnostic evidence of myocardial damage that differentiates them from the healthy subject of group $\mathrm{O}$, except for the presence of the parasite responsible for Chagas' disease.

However, the mechanism responsibly of changes of QRS loop energy and its morphology are not clearly explainable with our current data. The results presented in this work encourage the undertaking of deeper studies with the aim to correlate and better understand the relationship between the QRS loop parameters and Chagas' disease.

We conclude that the proposed analysis method of the VCG could be used to an additional diagnosis technique to evaluate the degree of myocardial damage in chronic chagasic patients. Finally, further investigations should be carried out to examine the proposed parameters in larger populations and to establish their prognostic value in a patients' classification scheme.

\section{ACKNOWLEDGEMENTS}

This work has been supported by grants from Consejo Nacional de Investigaciones Científicas y Técnicas and
Universidad Nacional de San Juan, both institutions from Argentina, and by Ministerio de Ciencia e Innovación de España (TEC2007-68076-C02-01). The authors thank Dr. Pedro Gomis for the Chagas' Database used in this study. The work of Dr. Arini was also supported by a grant from Fundación Florencio Fiorini from Argentina.

\section{REFERENCES}

[1] World Health Organization (WHO). Report of the Scientific Working Group on Chagas Disease. (www.who.int/tdr/diseases/chagas/ swg_chagas.htm), 2005.

[2] S. Morris, H. Tanowitz, M. Wittner, J. Bilezikian. "Pathophysiological insights into the Cardiomyopathy of Chagas' disease," Circulation vol. 82, pp. 1900-9, 1990.

[3] A. Rassi (Jr), A. Rassi, W. Little. "Chagas' heart disease," Clin. Cardiol. vol. 23, pp. 883-9, 2000.

[4] S. Dubner, E. Schapachnik, A.R. Pérez Riera, E. Valero. "Chagas disease: State-of-the-art of diagnosis and management". Cardiol. J. vol. 15, pp. 493-504, 2008.

[5] A.R. Pérez Riera, A.H. Uchida, C. Ferreira Filho, A. Meneghini, C. Ferreira, E. Schapacknik, S. Dubner, P. Moffa. "Significance of vectorcardiogram in the cardiological diagnosis of the 21st century", Clin. Cardiol. vol. 30, pp. 319-323, 2007.

[6] R. Correa, E. Laciar, P. Arini, R. Jané. "Analysis of QRS loop changes in the beat-to-beat vectocardiogram of ischemic patients undergoing PTCA," Conf. Proc. IEEE Eng. Med. Biol. Soc. pp. 1750-1753, 2009.

[7] A.L. Ribeiro, M.O. Rocha. "Indeterminate form of Chagas disease: Considerations about diagnosis and prognosis", Rev. Soc. Bras. Med. Trop. vol. 31, pp. 301-314, 1998.

[8] G. Breithardt, M.E. Cain, N. El-Sherif, N.C. Flowers, V. Hombach, M. Janse, M.B. Simson, G. Steinbeck. "Standards for analysis of ventricular late potentials using high-resolution or signal-averaged electrocardiography," Circulation vol. 83, pp. 1481-1488, 1991.

[9] F. Mora, P. Gomis, G. Passariello. "Señales electrocardiográficas de alta resolución en Chagas-El proyecto SEARCH," Acta Científica Venezolana vol.50, pp.187-194, 1999.

[10] J. Pan and W.J. Tompkins, "A real-time QRS detection algorithm," IEEE Trans. Biomed. Eng., vol. 32, pp. 230-236, 1985.

[11] L. Sörnmo, "Vectocardiographic Loop Alignment and Morphologic Beat-to-Beat Variability," IEEE Trans. Biomed. Eng., vol.45, no.12, pp. 1401-1413, 1998.

[12] K. S. Arun, T. S. Huang and S. D. "Blostein, Least-squares fitting of two 3-D point sets," IEEE Trans. Patt. Anal. Mach. Intell., vol PAMI9, no. 5, pp. 698-700, 1987.

[13] P. Gannedahls, S. Odeberg, O. Ljungqvist, A. Sollevi. "Vectorcardiographic changes during laparoscopic cholecystectomy may mimic signs of myocardial ischaemia," Acta Anaesthesiol Scand. vol. 41, pp. 1187-1192, 1997.

[14] C.B. Barber, D.P. Dobkin, H.T. Huhdanpaa. "The quick hull algorithm for convex hulls," ACM Trans Math Softw. vol. 22(4), pp. 469-483, 1996.

[15] Z. Kudaiberdiev. "Vectorcardiographic assessment of acute hypoxia effects in pulmonary hypertension due to chronic bronchitis," Anadolu Kardiyol Derg. vol. 7(1), pp. 198-200, 2007.

[16] J.P. Moraes-Filho, T.A. Moraes, V.N. Felix,A. C. Pereira-Barreto, Bettarello. "Esophageal Manometry and Vectorcardiography study of asymptomatic patients with chagas disease", Rev. Inst. Med. Trop. Sâo Paulo vol. 30(6), pp. 406-410, 1988.

[17] E. Laciar, R. Jané, D.H. Brooks. "Evaluation of Myocardial Damage in Chagasic Patients from the Signal-Averaged and Beat-to-Beat Analysis of the High Resolution Electrocardiogram," Comp. Cardiol. vol. 33, pp. 25-28, 2006.

[18] E. Pueyo, E. Laciar, E. Anzuola, P. Laguna, R. Jané. "Assessment of myocardial damage in chronic chagasic patients using QRS slopes", Comp. Cardiol., vol. 34, pp. 725-728, 2007. 